\title{
Análise de Tráfego para a Integração de Redes Sem Fio WiMax/WiFi: uma Abordagem Usando Cadeia de Markov
}

\author{
W. A. Gabler, G. H.S. Carvalho, C. R. L. Francês, J. C. W. A. Costa, S. V. Carvalho.
}

\begin{abstract}
Resumo-Neste trabalho é proposto um Controle de Admissão de Chamadas (CAC) com múltiplos thresholds para integração de redes WiMax e WiFi. Para a análise do modelo proposto são apresentados dois estudos. $\mathrm{O}$ primeiro compara o CAC proposto com o Compartilhamento Total. O segundo apresenta uma comparação do CAC para diferentes configurações de thresholds. Para a avaliação do esquema proposto é utilizada uma cadeia de Markov a tempo contínuo.
\end{abstract}

Palavras-Chave - WiMax, WiFi, Cadeias de Markov, Controle de Admissão de Chamadas.

Abstract-In this work it is proposed a Call Admission Control with multiples thresholds for WiMax and WiFi networks integration. To analyze the proposed model it is presented two studies. The first is a comparative study with the Complete Sharing scheme. The second shows a comparison of the CAC for different threshold settings. For the evaluation of the proposed scheme it is used a continous-time markov chain.

Keywords-WiMax, WiFi, Markov Chain, Call Admission Control.

\section{INTRODUÇÃO}

Um grande desafio, atualmente, consiste em fornecer conectividade a grandes distâncias e com altas taxas de transmissão. O WiMax (Worldwide Interoperability for Microwave Access), padrão IEEE 802.16, permite a criação de WMANs (Wireless Metropolitan Area Networks); no entanto, ainda oferece um custo proibitivo a usuários domésticos. As WLANs (Wireless Local Area Networks) têm sido uma boa opção de acesso para usuários finais através de hotspots WiFi (Wireless Fidelity); contudo, apresentam uma área de alcance limitada. A integração destas duas tecnologias vem sendo estudada no intuito de se obter uma maior área de cobertura, aumentando o número de usuários e aproveitando os equipamentos em uso já consolidados no mercado. Segundo [1] o uso de um backbone IEEE 802.16e/WiMax para conectar hotspots WiFi à Internet é menos custoso do que implantar infra-estrutura cabeada, além de possibilitar a implementação de aplicações de sistema de transporte inteligente (ITS).

\section{A. Trabalhos relacionados}

Segundo [2], um esquema de Controle de Admissão de Chamadas (CAC) objetiva manter QoS para diferentes chama-

Wellington de Araújo Gabler, Glaucio Haroldo Silva de Carvalho,Carlos Renato Lisboa Francês, João Crisóstomo Weyl A. Costa, Programa de PósGraduação em Engenharia Elétrica, Universidade Federal do Pará, Belém, Brasil, E-mails: \{gabler,ghsc,rfrances, jweyl\}@ufpa.br. Solon Venâncio de Carvalho, Laboratório Associado de Computação e Matemática Aplicada, Instituto Nacional de Pesquisa Espaciais, São José dos Campos, Brasil,Emails:solon@lac.inpe.br. das (ou usuários) no nível desejado, limitando o número de novas chamadas no sistema. O CAC proposto em [3] baseiase na utilização de Lógica Fuzzy e Algoritmos Genéticos resultando em dois esquemas: Fuzzy QoS Controller FQC e Fuzzy Congestion Controller FCC que juntos compõe o Fuzzy Admission Control for Multimedia Applications MFAC o qual considera vários parâmetros como: vazão, atraso, probabilidade de perda, etc. relevantes para aplicações multimídia. Em [4] é proposto um CAC que combina Lógica Fuzzy e Redes Neurais utilizando a capacidade aprendizado desta para reduzir os erros de decisão das políticas convencionais.

Em [5] é proposta uma política de CAC e um esquema de alocação de recursos que permite que provedores de serviço gerenciem a largura de banda de uma célula WiMax entre redes WiFi e a sua Subscriber Station (SS). Esse esquema segue a idéia de multi-threshold que tem sido estudada recentemente em redes celulares [6]-[8]. Em [1] são discutidas questões relacionadas à adaptação de protocolos, qualidade de serviços (QoS) e alocação de recursos em uma rede integrada WiMax/WiFi.

Outro trabalho relacionado pode ser consultado em [9] onde são propostos dois esquemas, considerando tráfego de alta e baixa prioridade: o primeiro utiliza somente um buffer para as duas classes de pacotes, sendo que, quando a ocupação do buffer é menor que o threshold utilizado, ambos os tipos de pacote são admitidos, caso contrário, somente os pacotes de alta prioridade; no segundo esquema, são utilizados dois buffers de forma que os pacotes que chegam são colocados em duas filas com prioridades diferentes. Nesse esquema, os pacotes posicionados na fila de baixa prioridade são processados somente se a fila de alta prioridade estiver vazia. Adicionalmente o buffer de baixa prioridade é dividido, utilizando-se um threshold, em duas áreas, uma que suporta apenas pacotes de baixa prioridade e outra que suporta pacotes de ambas as classes. Quando o buffer de alta prioridade está cheio, pacotes dessa classe podem ser adicionados no de baixa prioridade visando QoS.

Neste trabalho é proposto um modelo de CAC multithreshold no qual são levadas em consideração as classes de QoS estabelecidas no WiMax (UGS, rtPS, nrtPS, BE), juntamente com tráfego WiFi em tempo real e melhor esforço. $\mathrm{O}$ CAC proposto atribui uma maior prioridade ao tráfego WLAN e atende as diferentes classes de serviço na seguinte ordem de prioridade: WiFi em tempo real, UGS, PS (rtPS e nrtPS), WiFi e WiMax de melhor esforço. Nota-se que o modelo proposto privilegia o tráfego em tempo real partindo 
da WLAN, o que é justificado pela ausência de um suporte de QoS no padrão IEEE 802.11. O modelo utiliza thresholds para alocação de recursos para as classes de serviço estudadas, garantindo, assim, níveis de prioridade na alocação desses recursos. Para a viabilização da avaliação de desempenho do esquema proposto, é utilizada uma cadeia de Markov a tempo contínuo como abordagem para modelagem de sua dinâmica.

O trabalho é organizado como segue. Na seção 2 são feitas considerações sobre as redes WiMax e WiFi, assim como, aborda-se aspectos da integração dessas redes. Na seção 3 são feitas considerações sobre o Controle de Admissão proposto. Nas seções 4 e 5 são abordados o tráfego e a modelagem do sistema. Os resultados são apresentados na seção 6 e na seção 7 são feitas as conclusões do trabalho.

\section{Redes Sem Fio WiMax E WiFi}

\section{A. WiMax}

A tecnologia IEEE 802.16 também denominada WiMax, define um padrão para equipamentos utilizados na implementação de redes sem fio metropolitanas de banda larga. O padrão permite que a rede seja estruturada de duas maneiras: ponto a ponto - ligação entre duas BSs (Base Stations); e ponto multiponto, quando uma BS está ligada a mais de uma SS. Na camada física, como apresentado em [10], a interface aérea do IEEE 802.16 pode operar na faixa de 10-66 $\mathrm{GHz}$ (IEEE 802.16) ou 2-11 GHz (IEEE 802.16a) e suporta taxas de transmissão na faixa de $32 \mathrm{Mbit} / \mathrm{s}$ a $120 \mathrm{Mbit} / \mathrm{s}$, dependendo da largura de banda de operação (20, 25 ou 28 $\mathrm{MHz}$ ), da modulação e do esquema de codificação. O padrão suporta operação com linha de visada (LOS) e sem linha de visada (NLOS). Na camada de acesso ao meio (MAC), é usado um protocolo orientado a conexão. Sendo que uma SS pode solicitar largura de banda a BS. O padrão IEEE 802.16 suporta tanto FDD (Frequency Division Duplex) quanto TDD (Time Division Duplex). O suporte a QoS é definido por cinco tipos de fluxo de serviço, cada um com diferentes requisitos: UGS (Unsolicited Grant Service); rtPS (real-time Polling Service); nrtPS (non-real-time Polling Service) e BE (Best Effort Service). Segundo [11], existem muitas vantagens em sistemas baseados em IEEE 802.16, por exemplo, a habilidade de prover serviço, mesmo em áreas sem acesso por redes infraestruturadas, e a habilidade de superar outras limitações das redes infra-estruturadas. As redes WiMAX tem como outra vantagem seu grande raio de alcance, sendo possível utilizálas como rede metropolitana.

\section{B. WiFi}

As WLANs possibilitam um meio de conexão com altas taxas para diversos usuários; contudo, sua maior limitação reside no curto raio de alcance. Segundo [10], implementa dois tipos de arquitetura: infra-estruturada, na qual um AP (Access Point) conectado a uma rede de distribuição concentra o tráfego das estações de trabalho wireless; e topologia ad hoc na qual pelo menos duas estações se comunicam diretamente sem necessidade de uma estrutura preexistente. O padrão IEEE 802.11 utiliza frequências na faixa não licenciada de $2.4 \mathrm{GHz}$ e especifica taxas de dados de $1 \mathrm{Mbit} / \mathrm{s}$ e $2 \mathrm{Mbit} / \mathrm{s}$ para três diferentes camadas físicas: DSSS (direct sequence spread spectrum), FHSS (frequency hopping spread spectrum) e IR (infravermelho). Padrão IEEE 802.11a especifica taxas de 6 Mbit/s a $54 \mathrm{Mbit} / \mathrm{s}$ a $5 \mathrm{GHz}$, enquanto que padrão IEEE $802.11 \mathrm{~b}$ fornece taxas superiores a $11 \mathrm{Mbit} / \mathrm{s}$ a $2.4 \mathrm{GHz}$. O protocolo DCF (distribution coordination function) juntamente com CSMA/CA (carrier sense multiple access with collision avoidance) disciplinam o acesso à camada física. No padrão IEEE 802.11e são definidos aspectos de QoS.

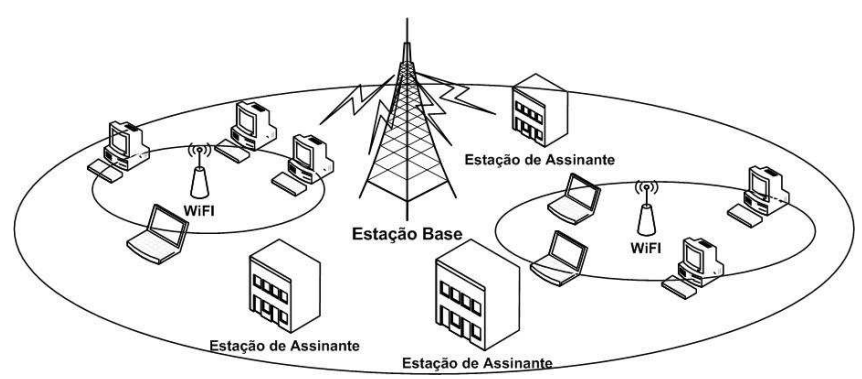

Fig. 1. Rede WiMax/Wifi fortemente acoplada

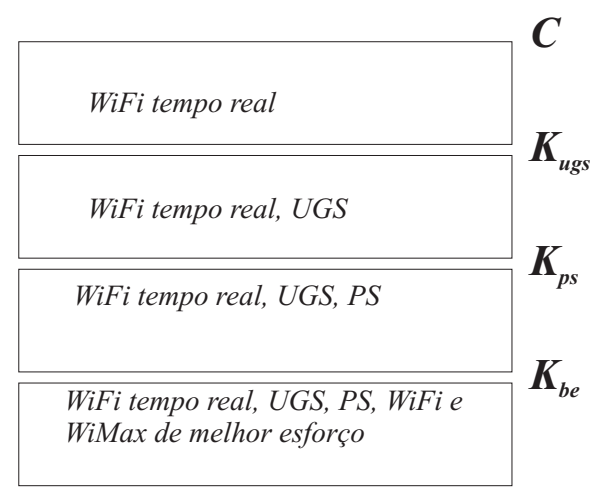

Fig. 2. Esquema de CAC proposto para integração WiMax/WiFi

\section{Considerações sobre a Integração das Redes Sem Fio WiMax e WiFi}

A grande vantagem na integração WiMax/WiFi reside em combinar suas vantagens e oferecer uma estrutura de comunicação de dados a um custo aceitável, oferecendo mapeamento de QoS/handoff vertical, do usuário. Na literatura observam-se duas abordagens para a integração das redes WLAN/WMAN: fracamente e fortemente acoplada. Na arquitetura fracamente acoplada poucas mudanças são necessárias em ambas as redes. Estas são ligadas em nível de camada IP permanecendo o tráfego de ambas separado, e as redes interligadas por meio de gateways. $\mathrm{Na}$ arquitetura fortemente acoplada, uma rede tenta emular o funcionamento da outra. O tráfego da rede WiFi passa pela rede WiMax que atua como uma rede de distribuição. Nesse caso o QoS se torna um fator crítico, pois o tráfego da WLAN concorre com as demais estações da WMAN.

A proposta desse trabalho é estudar um sistema fortemente acoplado, tendo em vista que sua integração possibilita diversos tipos de aplicações. Outro fator importante consiste no 
TABELA I

TABELA DE Estados $\phi=(i, j)$.

\begin{tabular}{llll}
\hline Próximo Estado & Condição & Taxa & Evento \\
\hline$(i, j+1)$ & $i+j<K_{b e}$ & $\lambda_{w f b e}+\lambda_{w x b e}$ & Chegada de uma chamada da classe de melhor esforço \\
$(i+1, j)$ & $i+j<K_{p s}$ & $\lambda_{w f}+\lambda_{u g s}+\lambda_{p s}$ & Chegada de uma chamada da classe WiFi, UGS ou PS \\
$(i+1, j)$ & $K_{p s} \leq i+j<K_{u g s}$ & $\lambda_{w f}+\lambda_{u g s}$ & Chegada de uma chamada da classe WiFi ou UGS \\
$(i+1, j)$ & $K_{u g s} \leq i+j<C$ & $\lambda_{w f}$ & Chegada de uma chamada da classe WiFi \\
$(i-1, j)$ & $i>0$ & $i \mu_{t r}$ & Partida de uma chamada da classe WiFi, UGS ou PS \\
$(i, j-1)$ & $j>0$ & $j \mu_{b e}$ & Partida de uma chamada de classe de melhor esforço \\
\hline
\end{tabular}

uso dos recursos das redes em sistemas fortemente acoplados, nos quais, o controle dos recursos pode ser realizado pela BS WiMax, nesse caso, existindo uma disputa pelos recursos entre o tráfego proveniente das WLANs e das SSs.

Duas políticas para controle de recursos podem ser: Compartilhamento Total (CT) a qual permite que todos os tipos de tráfego tenham o mesmo acesso a largura de banda disponível na BS; e Particionamento Completo (PC), a qual divide a largura de banda em partições, cada uma reservada para um tipo de tráfego. Neste trabalho, adotamos uma política que consiste no meio termo entre as apresentadas, denominada Multi-Threshold (MT), [2], [7], [8], descrita a seguir.

\section{CONSIDERAÇÕES SOBRE O CAC}

Neste trabalho, considera-se que a rede WiMax provê o acesso sem fio através de sua BS como pode ser observado na Fig.1. Toda a largura de banda do enlace WiMax é compartilhada entre as suas SS e as redes locais sem fio WiFi. Nesse sentido, o sistema em análise consiste do enlace de rádio WiMax, no qual seus $C$ canais de rádio são, de acordo com a Fig.2, divididos em quatro zonas de admissão pelos thresholds: $K_{u g s}, K_{p s}$ e $K_{b e}$. Assim, uma chamada da classe de melhor esforço (WiMax ou WiFi) somente é aceita pelo CAC se a ocupação dos recursos de rádio no enlace for menor que o threshold $K_{b e}$. Analogamente, uma chamada da classe PS (rtPS ou nrtPS) somente será aceita se a ocupação dos recursos de rádio for menor que o threshold $K_{p s}$. Uma chamada da classe de serviço UGS será aceita somente se a ocupação dos recursos de rádio for menor que o threshold $K_{u g s}$. Por fim, uma chamada em tempo real oriunda de uma rede local WiFi será aceita enquanto houver recursos disponíveis. Nota-se que a política adotada pelo CAC privilegia as chamadas em tempo real WiFi em relação às demais classes de serviço da rede. Essa política se justifica pelo fato do padrão IEEE 802.11 ( $a$, $b$ e $g$ ) não suportar QoS. Desse modo, ao liberar todos os canais de rádio para a chamada em tempo real WiFi, o CAC tenta mitigar a ausência de um suporte à QoS dentro da rede local WiFi.

\section{CONSIDERAÇÕES SOBRE O TRÁfEGo}

Para a confecção do modelo de Markov, dividiu-se as classes de serviço em dois grandes grupos denominados por: alta e baixa prioridade. Dentro do primeiro grupo estão as chamadas das classes de serviço: WiFi em tempo real, chamada UGS, chamada PS (rtPS e nrtPS). No segundo grupo estão presentes as chamadas de melhor esforço WiMax e WiFi. As chegadas das chamadas WiFi em tempo real, UGS, PS (rtPS e nrtPS), WiFi e WiMax de melhor esforço seguem processos de Poisson mutuamente independentes com taxas média dadas por $\lambda_{w f}, \lambda_{u g s}, \lambda_{p s}, \lambda_{w f b e}$ e $\lambda_{w x b e}$, respectivamente. Por simplicidade, considerou-se que todo fluxo de serviço rtPS e nrtPS está incluído em $\lambda_{p s}$.

O tempo de serviço de uma chamada WiFi em tempo real, UGS, rtPS e nrtPS são variáveis aleatórias distribuídas exponencialmente com valores médios dados por $T_{w f}=1 / \mu_{w f}$, $T_{u g s}=1 / \mu_{u g s}, T_{r t P S}=1 / \mu_{r t P S}$ e $T_{n r t P S}=1 / \mu_{n r t P S}$. Como resultado, o tempo de retenção de canal para as chamadas desse grupo é também distribuído exponencialmente com média $T_{t r}=1 / \mu_{t r}=1 /\left(\mu_{w f}+\mu_{u g s}+\mu_{r t P S}+\mu_{n r t P S}\right)$. Para o serviço de melhor esforço, grupo de baixa prioridade, não se faz distinção entre as chamadas vindas de redes locais WiFi ou de SS WiMax, [5], assim, considera-se que o tempo de serviço de uma chamada melhor esforço é uma variável aleatória exponencialmente distribuída com média $T_{b e}=1 / \mu_{b e}$.

\section{Modelagem}

Para modelar o esquema de CAC proposto usou-se uma cadeia de Markov a tempo contínuo com espaço de estados definido como:

$$
\phi=\left\{(i, j) / 0 \leq i \leq C, 0 \leq j \leq K_{b e}\right\}
$$

onde $i$ é o número de chamadas em tempo real das classes de serviço WiFi em tempo real, UGS e PS; $j$ é o número de chamadas da classe de melhor esforço. A Tabela I apresenta o modelo markoviano destacando os eventos que norteiam a dinâmica do sistema, com suas condições e taxas médias.

Para quantificar o desempenho do sistema, utilizou-se como critério de avaliação as métricas apresentadas nas equações de (2) até (7). A probabilidade de bloqueio de uma chamada de melhor esforço, denotada por $P_{b e}$, é definida como a probabilidade de uma chamada dessa classe solicitar acesso à rede e encontrar a ocupação dos recursos de rádio maior ou igual a $K_{b e}$. Seguindo uma idéia similar, com ressalva apenas em relação ao valor do threshold, têm-se nas Eq.(3), Eq.(4) e Eq.(5) as probabilidades de bloqueio da classe PS, UGS e WiFi de tempo real, respectivamente.

$$
P_{b e}=\sum_{i+j \geq K_{b e}}^{C} \pi(i, j),
$$




$$
\begin{gathered}
P_{p s}=\sum_{i+j \geq K_{p s}}^{C} \pi(i, j), \\
P_{u g s}=\sum_{i+j \geq K_{u g s}}^{C} \pi(i, j), \\
P_{w f}=\sum_{i+j=C} \pi(i, j),
\end{gathered}
$$

onde $\pi(i, j)$ é a distribuição do estado de equilíbrio da cadeia de Markov a tempo contínuo. Na Eq.(6) tem-se a utilização total dos recursos de rádio do enlace WiMax, que é definida como a relação entre o número médio de canais ocupados pelo número total de canais.

$$
\eta=\frac{\sum_{i>0}^{C} \sum_{j>0}^{K_{b e}}(i+j) \pi(i, j)}{C}
$$

O último critério usado neste trabalho é a função recompensa do sistema, definida na Eq.(7). Essa medida tem um papel fundamental na avaliação de desempenho por possibilitar analisar como o ajuste dos valores dos diferentes thresholds impactam no desempenho total do sistema.

$R=\eta-\left(\alpha_{w f} \log _{10}^{P_{w f}}+\alpha_{u g s} \log _{10}^{P_{u g s}}+\alpha_{p s} \log _{10}^{P_{p s}}+\alpha_{b e} \log _{10}^{P_{b e}}\right)$

em que os pesos $\alpha_{w f}+\alpha_{u g s}+\alpha_{p s}+\alpha_{b e}=1$.

\section{RESUltados}

Nesta seção são apresentados os resultados da avaliação do CAC proposto, comparando-o com um modelo de compartilhamento total, e comparando diferentes valores de threshold. Para estudo do modelo proposto, foram adotados os valores de taxas de chegada e tempos de serviço na Tabela II. O tráfego oferecido representa a soma dos tráfegos de alta e baixa prioridade. $\mathrm{O}$ tráfego de baixa prioridade se manteve fixo, enquanto que, o tráfego de alta prioridade foi aumentado em $50 \%$. Na comparação entre o CAC proposto e um modelo CT, os valores definidos para os thresholds foram: $K_{u g s}=28$, $K_{p s}=27$ e $K_{b e}=26$. O modelo CT foi obtido com os seguintes thresholds: $K_{u g s}=K_{p s}=K_{b e}=C=30$. Para a função recompensa, foram adotados os seguintes pesos: $\alpha_{w f}=0.4, \alpha_{u g s}=0.3, \alpha_{p s}=0.2$ e $\alpha_{b e}=0.1$, para os diferentes tipos de tráfego, visando possibilitar uma análise do desempenho total do sistema.

\section{A. Comparação do CAC Proposto com Compartilhamento Total}

Observa-se, na Fig.3, que em uma comparação entre um cenário com $\mathrm{CT}$, no qual todas as classes de serviço possuem a mesma probabilidade de bloqueio, e o modelo proposto, o tráfego WiFi é privilegiado com a menor probabilidade de bloqueio, garantindo melhor QoS para o tráfego proveniente da WLAN. No modelo multi-threshold, todas as classes, com exceção do melhor esforço, possuem uma probabilidade de
TABELA II

PARAMETRIZAÇÃo.

\begin{tabular}{|l||c|c|}
\hline Serviço & Chamada/h & Tempo(seg) \\
\hline \hline UGS & 20 & 1500 \\
\hline rtPS & 4.8 & 1500 \\
\hline nrtPS & 6 & 3600 \\
\hline WiFi(Tempo Real) & 10 & 1800 \\
\hline WiFi (Melhor Esforço) & 10 & 1800 \\
\hline WiMax (Melhor Esforço) & 40 & 1800 \\
\hline
\end{tabular}

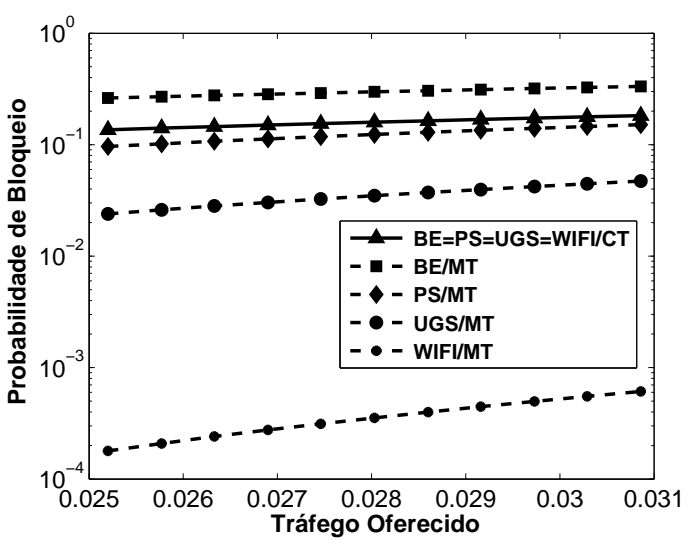

Fig. 3. Probabilidade de bloqueio CT e MT

bloqueio inferior às probabilidades de bloqueio obtidas com o compartilhamento total.

A utilização do sistema, Fig.4, é maior quando todos os recursos são compartilhados, isso ocorre pois não há reserva de recursos para uma classe em particular, isso permite que todas as classes aloquem recursos enquanto estes estiverem disponíveis; contudo, a recompensa é maior no modelo MT, como pode ser observado na Fig.5, devido ao modelo MT privilegiar as classes de maior peso.

\section{B. Avaliação do CAC Proposto para diferentes valores de threshold}

Em uma outra análise foram avaliados três diferentes conjuntos de valores para thresholds:

$$
\begin{aligned}
& -K_{u g s}=28, K_{p s}=27 \text { e } K_{b e}=26 ; \\
& \text { - } K_{u g s}=29, K_{p s}=27 \text { e } K_{b e}=25 ; \\
& \text { - } K_{u g s}=29, K_{p s}=28 \text { e } K_{b e}=27 .
\end{aligned}
$$

Na Fig.6 é apresentada a probabilidade de bloqueio do tráfego WiFi. Percebe-se que quanto mais canais são disponibilizados exclusivamente para o tráfego WiFi menor a sua probabilidade de bloqueio. Na Fig.7 é apresentada probabilidade de bloqueio do tráfego UGS. Observa-se que a melhor situação ocorre quando a diferença entre os thresholds é maior. Por exemplo: $K_{u g s}=29, K_{p s}=27$ e $K_{b e}=25$. Isso mostra que quando há mais recursos disponíveis para a classe, ou seja, o intervalo entre os valores é maior, a probabilidade de bloqueio é menor.

Semelhante ao caso anterior, para o tráfego PS, como pode ser visto na Fig.8, a melhor configuração foi obtida com a maior diferença entre os thresholds: $K_{u g s}=29, K_{p s}=27 \mathrm{e}$ 


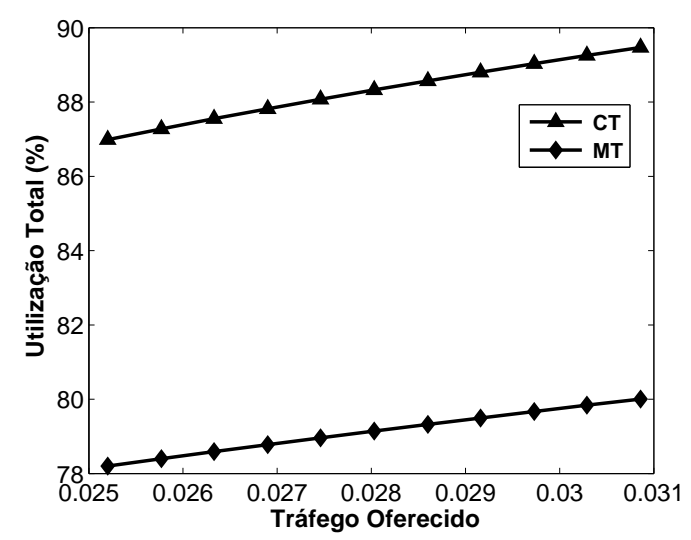

Fig. 4. Utilização CT e MT

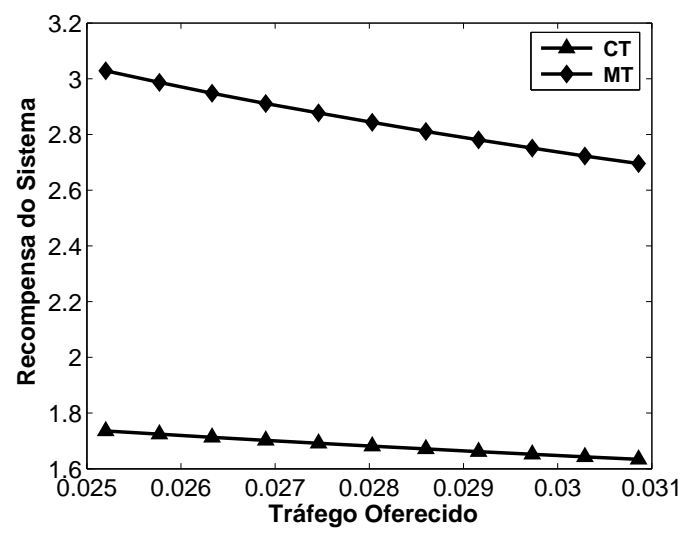

Fig. 5. Recompensa CT e MT

$K_{b e}=25$, com o aumento do número de recursos, menor a probabilidade de bloqueio.

O tráfego BE, Fig.9, apresenta menor probabilidade de bloqueio para: $K_{u g s}=29, K_{p s}=28$ e $K_{b e}=27$ e maior probabilidade de bloqueio para $K_{u g s}=29, K_{p s}=27 \mathrm{e}$ $K_{b e}=25$, ou seja, quanto menos recursos disponíveis para esse tipo de tráfego, maior a sua probabilidade de bloqueio.

De acordo com a Fig.10, a utilização do sistema é menor quando mais recursos são reservados para cada serviço individualmente e é melhor quanto mais o sistema dispõe de recursos comuns. Por exemplo: $K_{u g s}=29, K_{p s}=27$ e $K_{b e}=25$; e, $K_{u g s}=29, K_{p s}=28$ e $K_{b e}=27$ respectivamente.

A Fig.11 ressalta que o sistema tem uma maior recompensa quando há uma quantidade maior de recursos reservados individualmente para cada tipo de tráfego. Por exemplo: $K_{\text {ugs }}=$ $29, K_{p s}=27$ e $K_{b e}=25$.

\section{CONCLUSÃO}

Nesse trabalho foi proposto um CAC com múltiplos thresholds para a integração WiFi e WiMax. Uma breve descrição das tecnologias foi apresentada, bem como, uma política de admissão baseada em Multi-Thresholds, além de considerações sobre o controle de admissão, sobre o tráfego e sua modelagem. A partir da análise dos resultados obtidos, concluiu-se que o modelo consegue prover garantia de qualidade de serviço para classes individuais. Foram apresentados

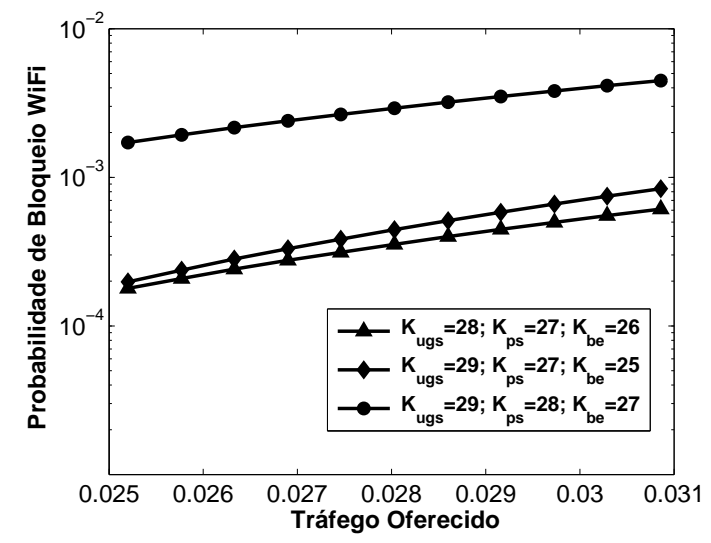

Fig. 6. Probabilidades de bloqueio WiFi

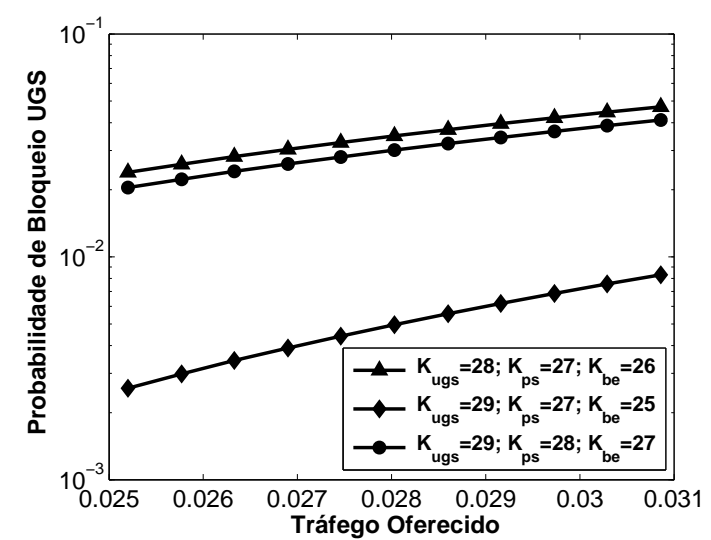

Fig. 7. Probabilidades de bloqueio UGS

resultados da avaliação do sistema obtidos através de Cadeia de Markov. Uma das dificuldades encontradas no modelo é o ajuste dos valores dos thresholds. Para resolver esse problema, está-se considerando utilizar o processo markoviano de decisão para o ajuste ótimo desses valores de acordo com o perfil de QoS de cada classe. Esse estudo será alvo de trabalhos futuros. O modelo proposto pode ser facilmente extendido para lidar com uma diferenciação entre o tráfego rtPS e nrtPS pela criação de mais um threshold.

\section{AGRADECIMENTOS}

Os autores agradecem a CAPES (Coordenação de Aperfeiçoamento Pessoal) pelo suporte financeiro ao projeto 0226/05-0 dentro do programa PROCAD (Programa de Cooperação Acadêmica).

\section{REFERÊNCIAS}

[1] D. Niyato, E. Hossain. Wireless broadband access: Wimax and beyond integration of wimax and wifi:optimal pricing for bandwidth sharing. IEEE Communications Magazine, 45(5):140-146, May 2007.

[2] D. Niyato, E. Hossain, Call Admission Control for QoS Provisioning in 4G Wireless Network: Issues and Approaches, IEEE Network, No. 4, pp. 5-11, Setembro/Outubro, 2005.

[3] M. Ikeda, L. Barolli, A. Koyama, A. Durresi, G. D. Marco, J. Iwashige. Performance evaluation of an intelligent CAC and routing framework for multimedia applications in broadband neworks. Journal of Computer and System Sciences, 72 : 1183-1200, Nov 2006. 


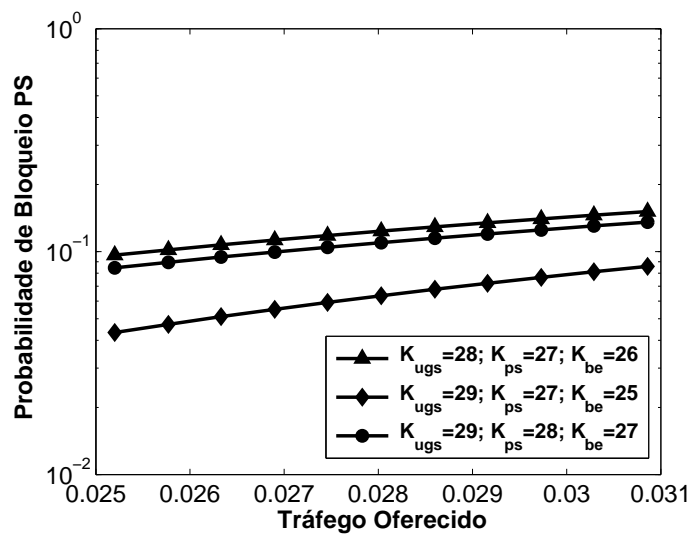

Fig. 8. Probabilidades de bloqueio PS

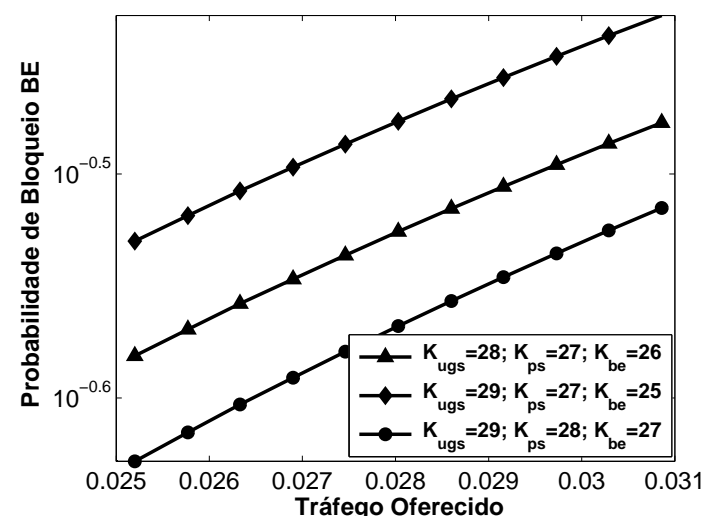

Fig. 9. Probabilidades de bloqueio BS

[4] R. Cheng, C. Chang. A QoS-Provisioning Neural Fuzzy Connection Admission Controller for Multimedia High-Speed Networks. IEEE/ACM Transactions 7(1): 111-121, Feb 1999.

[5] T. Yahiya, A. Beylot, G. Pujolle, Policy-based threshold for bandwidth reservation in wimax and wifi wireless networks. In ICWMC '07: Proceedings of the Third International Conference on Wireless and Mobile Communications, pp. 76.

[6] B. Li, L. Li, B. Li, K. M. Sivalingam, X.-R. Cao. Call admission control for voice/data integrated cellular networks: Performance analysis and comparative study. IEEE Journal on Selected Areas in Communications, 22(4):706-718, May 2004.

[7] S. Tang, W. Li. An adaptive bandwidth allocation scheme with preemptive priority for integrated voice/data mobile networks. IEEE Transaction on Wireless Communications, 5(10):2874-2886, October 2006.

[8] S. E. Ogbonmwan, W. Li. Multi-threshold bandwidth reservation scheme of an integrated voice/data wireless network. Elsevier J. Computer Communications, 29(9):1504-1515, May 2006.

[9] G. H. S. Carvalho, V. S. Martins, C. R. L. Francês, J. C. W. A. Costa, S. V. Carvalho. Performance analysis of multi-service wireless network: An approach integrating CAC, scheduling and buffer management. Computers and Electrical Engineering 34 (4): 346-356. Available online 18 October 2007.

[10] D. Niyato, E. Hossain, J. Diamond, IEEE 802.16/WiMAX-Based Broadband Wireless Access and It's Application for Telemedicine/EHealth Services. IEEE Wireless Comunications. Vol. 14, Issue 1, 2007 Page(s):72 - 83.

[11] D. Pareek, Understanding WiMAX: The business of WiMAX John Wiley \& Sons, England, 2006

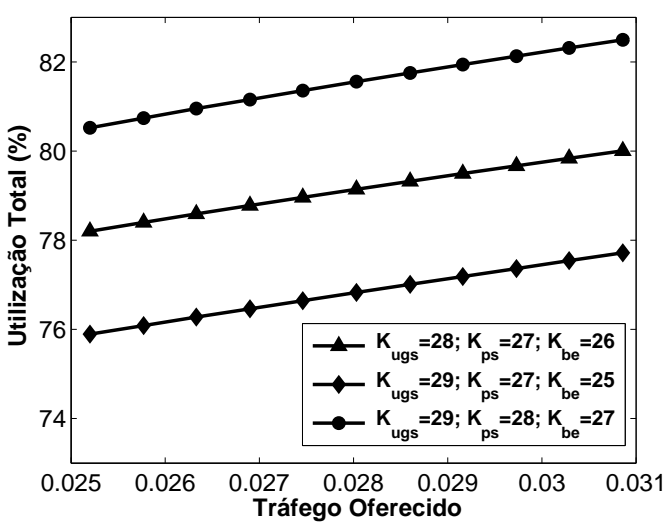

Fig. 10. Utilização total MT

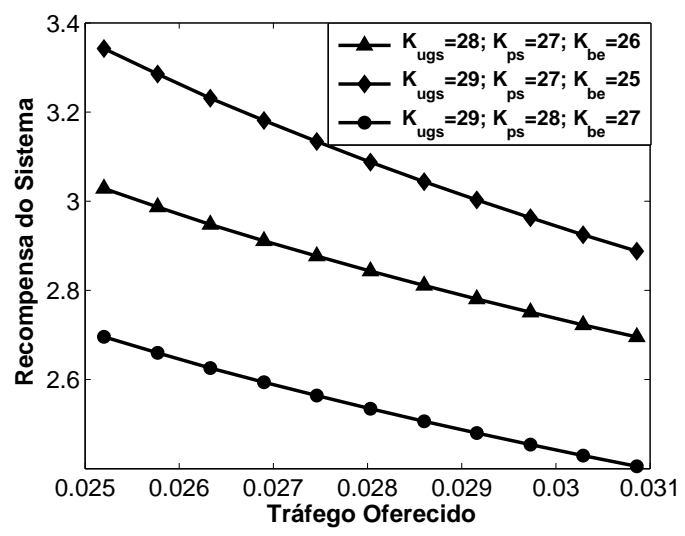

Fig. 11. Recompensa MT 